\title{
Ireland's Participation in the 47th International Mathematical Olympiad
}

\author{
RACHEL QUINLAN
}

The 47th International Mathematical Olympiad (IMO) took place in Slovenia from July 12 th to July 18th, 2006. It was my privilege to be the Irish leader at this event, and this is my account of the experience. It contains a factual description of this IMO and Ireland's participation in it based on my notes and recollection, and also some remarks and commentary. Opinions expressed in this document are my own.

The International Mathematical Olympiad is a contest in mathematical problem-solving. According to IMO rules, contestants must be under the age of 20 at the time of the contest and must never have been enrolled as full-time students in a third level educational institution. Each participating country may enter up to six contestants. The contest runs over two days, and on each day participants have 4 hours 30 minutes to tackle three problems. Each day's paper consists firstly of a difficult problem, then an even more difficult problem, and finally an incredibly difficult problem. From now on we will use the traditional IMO parlance for these grades of difficulty - easy, medium, and hard (respectively).

Problems are proposed by participating countries well in advance of each IMO, and the collection of submissions is whittled down by the Problem Selection Committee to a short-list of approximately 30 problems, listed under the headings of Algebra, Combinatorics, Geometry, and Number Theory. To be suitable for an IMO a problem must be challenging but must admit a solution without recourse to advanced mathematical theory not normally known to secondary school students. It must not be known to appear in the literature or in any past contests. A good problem will also have an aesthetic appeal, at least to mathematically inclined individuals. Composing suitable problems is an extremely difficult task. 
In the days leading up to the contest six problems are selected from the short-list by a jury consisting of the leaders from the participating countries. The term "leader" incidentally does not really imply a particular leadership role - it is the word used for that person from a country's delegation who sits on the jury. As well as the leader each country has a deputy leader who accompanies the students until the conclusion of the contest and then joins the leader for the important task of coordination - this is the assessment of the contestants' performance and assignment of marks. In the days leading up to the contest, the leaders and contestants are accommodated at different locations, as the leaders have knowledge of the problem short-list. Countries may also send one or more observers to accompany the leader or deputy leader and share the overall workload associated with these roles.

The Irish team in Slovenia consisted of

IRL 1 Galin Ganchev

IRL 2 Krzysztof Bryla

IRL 3 Anthony Walsh

IRL 4 Derek O'Brien

IRL 5 Stephen Dolan

IRL 6 Jamie Judd

The Irish deputy leader was Gordon Lessells of the University of Limerick, and Richard Watson of NUI Maynooth was the Irish observer, at the leaders' site.

\section{Team Selection and Preparation}

The selection contest for the Irish IMO team is the Irish Mathematical Olympiad (IrMO), which was held for the 19th time on May 6th this year. Contestants in the IrMO have normally participated in a mathematical enrichment programme through the period December/January to May at one of five centres around the country. These centres are located at NUI Galway, NUI Maynooth, UCC, UCD and UL, and the enrichment programmes are run on an entirely voluntary basis by members of staff of these and nearby institutions. Typically the enrichment programmes consist of regular lectures and problemsolving sessions as well as practice problems on which students are encouraged to work independently.

The IrMO involves two three-hour papers each consisting of five problems arranged in approximately increasing order of difficulty. 
Problem proposals are submitted by the instructors from the various enrichment programmes, and this year the contest problems were selected at a meeting at the University of Limerick in April. One aim in selecting the problems for an IrMO is to have a good mixture of easy and hard problems so that the strongest contestants should be challenged, while no participant should be discouraged by the experience. The hardest problems on the IrMO would be approaching the level of difficulty of a medium IMO problem, so that a strong performance in the IrMO would certainly indicate potential to succeed at the IMO.

This year's IrMO was marked according to the usual procedure: scripts at each centre are marked by the local staff according to marking schemes devised by the problem proposers. The best scripts from each centre were brought to a meeting at the University of Limerick, where they were inspected and compared before the final ranking was decided. The top six performers were invited to represent Ireland at the 47th IMO and fortunately all six were in a position to do so. The top ten contestants at the this year's IrMO (and their schools) were
1. Galin Ganchev
Castletroy College, Limerick
2. Krzysztof Bryla
St Benildus College, Dublin
3. Anthony Walsh
Carrigaline Community School
4. Derek O'Brien
Wesley College, Dublin
5. Stephen Dolan
Coláiste Colmcille, Ballyshannon,
Co. Donegal
6. Jamie Judd
St Gerard's, Bray, Co. Wicklow
7. Robin Tobin
St Aidan's CBS, Dublin
8. Patrick Forde
Coláiste an Spiorad Naomh, Bishopstown, Cork
9. Martin Cotton
Sligo Grammar School
10. Michael O'Rourke
Belvedere College, Dublin

A pre-IMO training camp, organised by Gordon Lessells, was held at the University of Limerick from July 3rd to 6th. The Limerick camp has become an annual fixture in the run up to Irish IMO participation. It is single-handedly organised every year by Gordon, with low-key efficiency and unfailing good humour. Participants in this year's camp included the team members as well as some other strong performers in the selection contest, who are eligible for future IMOs. Lectures and problem-solving sessions at the camp were 
given by local volunteers and visitors from the other centres. This year we were fortunate that former Irish Mathematical Olympians Mark Flanagan and Fiachra Knox were able to attend the camp and offer mathematical and moral support to the team members. The Limerick camp also included a very enjoyable reception in Plassey House at which the team members were welcomed by Professor Don Barry, acting president of the University of Limerick, and by Councillor Kathleen Leddin of Limerick City Council. On Tuesday July 4th I bid Gordon and the team farewell and left Limerick in order to prepare to depart for Slovenia early on Thursday morning. Gordon and the contestants would fly out from Dublin on the following Monday morning.

\section{Problem Selection}

I left Galway early on Thursday July 6th to catch a midday flight from Dublin to Frankfurt. I met Richard at the check-in desk, which was mercifully uncrowded. In fact we were lucky to be flying with Lufthansa as a union meeting was causing major disruption to all Aer Lingus flights from Dublin that morning. The World Cup was in full swing and there was plenty of evidence of that at Frankfurt Airport, where we had a couple of hours to kill before catching our connection to Ljubljana. At the departure gate in Frankfurt we met some other IMO-bound people including the New Zealand leader Michael Albert. I first met Michael in 2002 when he and the New Zealand team attended the camp in Limerick, en route to Scotland for the IMO. Upon arrival in Ljubljana we were warmly welcomed by IMO staff who informed us that the leaders would be accommodated in Portorož, a coastal resort town close to the Croatian border.

The drive from Ljubljana Airport to Portorož takes nearly two hours and it was midnight when we arrived, too late to collect our information packs and problem short-lists. We did discover from the notice board in the hotel lobby that the first jury meeting was scheduled for 9.00 next morning. After a long day's travelling we were grateful to retire to very pleasant and comfortable rooms.

Friday July 7th

The first jury meeting turned out to be a short affair as expected. We were welcomed by Gregor Dolinar who would prove to be an extremely capable and efficient jury chairperson. After some points of information we were dispatched to spend the day considering the 
short-listed problems. The jury would reconvene at 9.00 the next morning to begin work on the selection of contest problems. Richard and I, having just received the short-list that morning, went off separately to our sunny balconies to peruse it. We would meet for lunch at 1.00 and exchange notes then.

The short-list contained six algebra problems, eight combinatorics problems, ten geometry problems and seven number theory problems. It is usual that the selected problems would include at least one from each of these sublists, and in recent years it seems to have become conventional to include two geometry problems. I did not have time that morning to seriously engage with any of the geometry problems but I noted that there were some with a combinatorial flavour as well as plenty of the more traditional Euclidean geometry type. I spent more time thinking about the problems from the other three categories and was able to make progress with some of them. I was pleased to identify several problems that seemed possibly accessible to me (and thus hopefully to our students as well). Amongst the harder problems I liked the look of some of them although I cannot say that I considered them very deeply before the distribution of solutions.

I must point out that some of the other leaders and observers, having arrived earlier on the previous day, had enjoyed extra time to study the short-list. It is possible that the revelation at lunchtime that some had more insight than I into the problems can be attributed solely to this fact. But that is unlikely. United Kingdom observer Joseph Myers revealed to us at lunch that he had solved all the combinatorics problems and was now going through them a second time in order to create another swathe of solutions. Joseph is a formidable problem solver and was twice an IMO gold medallist, representing the UK. It was very pleasant to renew some old acquaintances from my only previous experience of an IMO, in Scotland in 2002. These included the Luxembourg leader, Charles Leytem, who had also attended the Limerick camp in 2002, and the United Kingdom leader Geoff Smith, a character known for witty repartee and colourful exposition of common sense.

Solutions to the short-listed problems were distributed later on Friday afternoon, and we spend the next few hours studying them. Of course it is far preferable to be able to form an impression of a problem without consulting a supplied solution, but in the time available it is simply impossible to do this for all the problems. 
Saturday July 8th

The jury assembled at 9.00 on Saturday to begin the task of problem selection. The first order of business was the expression of concerns about the suitability of any problems on any grounds. Problems C3 and N7 were quickly eliminated as closely related problems had recently appeared on two national contests. Concerns were also raised about Problem C4 which involved a standard theoretical construction that had been discussed with students by at least one leader during training. This problem was also eliminated after some discussion. It seemed to be generally accepted also that Problem C7 was not really suitable as it involved polyhedral geometry, a topic traditionally excluded from the unofficial IMO syllabus. Although this problem was not officially eliminated it was not seriously regarded as a contender for selection. It was noted that we were now left with a shortage of combinatorics problems - from the original seven there now remained the relatively easy $\mathrm{C} 1$, the medium problem $\mathrm{C} 2$ and the difficult problems C5 and C6.

The next task was the filling out of the "Difficulty and Beauty" forms. Leaders are invited to grade both the difficulty and aesthetic appeal of each problem as low, medium or high. Both of these qualities can only be reasonably assessed by a person who has seriously considered the problem, and we were advised to assess only those problems that we had studied. Richard and I filled out the form as best we could but like many other people we left plenty of blanks. The results of the difficulty and beauty contest were distributed at the next jury meeting. Naturally the easier problems had been rated by many more people than the most difficult ones, which take much longer to analyse. This survey, particularly the "difficulty" aspect of it, is a crude but useful measure as it enables the jury to roughly categorise problems as easy, medium or hard, and proceed with the selection.

The protocol in recent years has been to propose hard, easy or medium problems in pairs, and to conduct rounds of voting on the proposed pairs, eliminating the least popular pair after each round until only one remains. The two problems in this remaining pair are then deemed to be selected for the contest. It is possible to vote for more than one pair in each round although obviously pointless to vote for all of them. It is standard practice to begin with either the two easy problems or the two hard problems, and to leave the medium problems for the last phase of the selection. 
It was decided to employ this procedure as usual and to begin with the selection of the two hard problems, as it seemed that there was a wide array of attractive easy/medium problems to choose from. The process of nominating pairs of hard problems began, and after several efficient rounds of voting we had selected Problems A6 and G10. Problem G10 was an exceptionally difficult and attractive problem in combinatorial geometry, and Problem A6 was an inequality/optimisation problem with three variables and a surprising answer. It had the look of the sort of problem that Finbarr Holland might propose, and would later be revealed to be exactly that.

The next step was to choose two easy problems. Once again numerous pairs were nominated and after several elimination rounds Problems G1 and N1 were chosen. These were the two problems considered to be the easiest by the jury, and both were likeable problems. I was reasonably pleased with this selection as I considered that our students would be able to make some progress with both of these problems.

It was now time to select the two medium problems, and in fact these were almost selected by default. By now we had chosen one algebra problem, one number theory problem, and two geometry problems. It was clear that the medium problems must include one from the combinatorics list, and in fact $\mathrm{C} 2$ was the only reasonable candidate. Problems C3, C4 and C7 had already been eliminated, Problems C5 and C6 were considered by most people to be too difficult, as we had already chosen two very difficult problems in A6 and G10. Problem C1 was not very popular and was reckoned to be too easy, as we had chosen the two easiest problems on the short-list in G1 and N1.

This left one problem of medium difficulty to choose. Geometry problems were ruled out since we had two of them already. Problems left from the combinatorics list were out for the reasons outlined above. From the limited choice that remained, Problem N4 was selected after a few rounds of voting. This completed the problem selection, and since there was plenty of time for jury meetings left on Saturday we proceeded with the arrangement of problems in the two papers. The first decision to make was the assignment of the two hard problems as contest problems 3 (Day 1) and 6 (Day 2). Since G10 was considered to be the harder of the two it was assigned as Problem 6. Problem G1 immediately became Problem 1 in order 
to avoid having the two geometry problems on the same day. This left N1 as Problem 4, leaving only the two medium problems. Since C2 was considerably easier than N5 it was chosen as Problem 2. Although this meant that both number theory problems would be on Day 2, they were quite different in flavour.

We marvelled at the efficiency and smooth running of the problem selection machine, and went to a nearby bar to watch Portugal and Germany battle for third place in the World Cup.

\section{Sunday July 9th}

Despite the efficiency and smooth running, like some others I was not entirely satisfied with the selection process, and not convinced that we were ending up with the best contest possible from the extensive and interesting short-list. The problem selection algorithm has some significant strengths - it is efficient and systematic and limits the amount of time that can be spent on unfocussed and inconclusive waffle. With the brisk rounds of voting it terminates in quadratic time (the number of pairs of problems being a quadratic function of the number of problems). However its weaknesses seemed very clear - discussion tends to centre around the uninteresting consideration of which problems can sensibly be paired together as least as much as around the mathematical nature of the problems. A more serious drawback however in my opinion is that the progress of the algorithm eliminates lots of problems which never get a chance to be considered, and selects certain problems by default, also without consideration of their merits. This year for example, G10 was selected very early as an attractive very hard problem, and G1 was selected as a very easy problem. This meant that any geometry problem that could possibly be classified as medium was eliminated without consideration. On the other hand, Problem C2 was selected automatically, not because anybody particularly spoke up for it but because at some point it was the only reasonable choice. It seems to me that a better way to proceed might be to first select the two easy or the two hard problems, and then select a pair of problems from the two categories not yet represented. This would leave problems from all four subject areas still in contention for selection in the last pair. It is true that it might rule out certain easy or very hard problems, but the division of problems into difficulty levels is fuzzier than the division into subject areas, so there might be more room for manoeuvre. I was not at all unhappy with the outcome this year, and in particular I was pleased 
that we succeeded in choosing two hard problems, two easy ones and two of medium difficulty. It is quite possible for the process to produce three hard problems and three easy ones.

In any case the problems were selected now and the first item of business on Sunday morning was the meeting of the English language committee, whose task it is to produce an official written statement of the problems in English. Membership of the English language committee is open to all interested persons. It was chaired this year (as in recent years) by Geoff Smith, with Michael Albert acting as secretary. Many leaders turn up to this meeting simply to be entertained by the chairing technique, and not because they or their students have any interest in the English language version of the problems. Geoff's method of not causing lasting offence is to insult everybody the same amount and in hilarious style. Not even the Irish are spared.

Care is needed in preparing the official English language version, the objective being to produce statements that are concise, correct, and impossible to misinterpret. Imprecision can cause confusion among students but so also can excessive pedantry, so a balance must be found. Without much disagreement we produced a statement of the problems to everybody's satisfaction. This was duly approved by the jury after lunch, whereupon work commenced on the translations into the other official languages of French, German, Russian and Spanish. Trouble emerged during this work with the phrasing of Problem 2. The difficulty was already present in the English version but lurking in the background; the word "odd" was being used in two different ways. This was considered acceptable in the English version but caused problems in translation. After a long and intermittently heated discussion the matter was resolved by changing "odd" in one of its senses to "good".

The official language versions of the problems were approved by the jury in due course, and translations into all the requisite languages were then prepared. The problems of the 47th IMO were as follows.

\section{DAY 1: JULY 12TH}

(1) Let $A B C$ be a triangle with incentre $I$. A point $P$ in the interior of the triangle satisfies

$$
\angle P B A+\angle P C A=\angle P B C+\angle P C B .
$$


Show that $A P \geq A I$, and that equality holds if and only if $P=I$.

(2) Let $P$ be a regular 2006-gon. A diagonal of $P$ is called good if its endpoints divide the boundary of $P$ into two parts, each composed of an odd number of sides of $P$. The sides of $P$ are also called good.

Suppose $P$ has been dissected into triangles by 2003 diagonals, no two of which have a common point in the interior of $P$. Find the maximum number of isosceles triangles having two good sides that could appear in such a configuration.

(3) Determine the least real number $M$ such that the inequality

$\left|a b\left(a^{2}-b^{2}\right)+b c\left(b^{2}-c^{2}\right)+c a\left(c^{2}-a^{2}\right)\right| \leq M\left(a^{2}+b^{2}+c^{2}\right)^{2}$

holds for all real numbers $a, b$ and $c$.

\section{DAY 2: JULY 13TH}

4. Determine all pairs $(x, y)$ of integers such that

$$
1+2^{x}+2^{2 x+1}=y^{2} .
$$

5. Let $P(x)$ be a polynomial of degree $n>1$ with integer coefficients and let $k$ be a positive integer. Consider the polynomial $Q(x)=P(P(\ldots P(P(x)) \ldots))$, where $P$ occurs $k$ times. Prove that there are at most $n$ integers $t$ such that $Q(t)=t$.

6. Assign to each side $b$ of a convex polygon $P$ the maximum area of a triangle that has $b$ as a side and is contained in $P$. Show that the sum of the areas assigned to the sides of $P$ is at least twice the area of $P$.

\section{Monday July 10th}

The only remaining task for the jury before the contest was the approval of marking schemes for the six problems. This was completed on Monday morning. The procedure for agreement of marking schemes at the IMO is as follows. Each problem has a chief coordinator, who proposes a marking scheme and presents it to the jury. Discussion follows, after which the chief coordinators may revise their schemes. The revised schemes are later presented and approved. Designing a suitable marking scheme for an IMO problem is a difficult task. Marking schemes need to be detailed and decisive so that they can be applied consistently and fairly; however they are designed and 
approved in advance of the contest and cannot cover every possible eventuality.

\section{The Contest}

On Tuesday afternoon we travelled from Portorož to Ljubljana for the opening ceremony, which took place in the Grand Hotel Union. The leaders and leader observers sat in a balcony which runs around three sides of the hall, while the students and deputy leaders were seated on the main floor. The ceremony was very enjoyable, beginning with some short speeches and the unfurling of the IMO banner to mark the official opening of the event. The Slovenian national anthem was beautifully sung by a small choir, with an English translation shown on the screen.

After further musical performances came the parade of nations in which each team enjoyed a few seconds on the stage. We witnessed many different styles of parading, including a dramatic reenactment by the Italians of their match-winning penalty against Australia in the World Cup. The Irish team members behaved in an entirely civilised manner as we expected, and they appeared to be enjoying themselves. We were ushered out of the hall before the ceremony ended, so that interaction with students on the way out would be avoided. We waved to the team and to Gordon and walked the short distance to the University of Ljubljana where a reception had been arranged. The walk along the banks of the river Ljubljanica gave us our first glimpse of the beautiful and quiet city centre to which we would return a few days later.

The contest began at 9.00 on the next morning. During the first 30 minutes of each paper, contestants have the opportunity to direct queries about the problems to the jury. They do so by writing their question on a form which is relayed to leaders' site and displayed to the jury. The jury agrees on an answer which is then supplied by the leader of the student's country. The most popular answers are "No comment" or "Read the problem again" but occasionally a request for clarification can be answered in more detail. On this occasion the vast majority of queries concerned the configurations described in Problem 2 and were dealt with uncontroversially. There were no queries from the Irish students, which I interpreted as a good sign.

After an early lunch we headed off on a very enjoyable excursion. The first port of call was the tiny church of Hrastovlje which dates 
from the 12th century and whose interior is richly and beautifully decorated with frescoes. According to the internet the frescoes were completed in the late 15th century and the church was later fortified with high outer walls. We then proceeded to the spectacular Postojna cave, which for me was the (non-mathematical) highlight of the whole trip. This enormous cave is one of several in the area and is the most easily accessible to tourists. Our visit involved a $3 \mathrm{~km}$ train ride from the exterior into the heart of the cave, followed by a guided walk of about $1.5 \mathrm{~km}$ and a train journey back out. We were told that this trip only covers a fraction of the entire Postojna cave system, which made the whole experience even more amazing. The final phase of our excursion was a visit to the picturesque Predjama Castle, located close to Postojna.

As the bus headed back towards Portorož we were informed that the morning's scripts were waiting for us at the IMO office. This news was greeted with mixed feelings by most of us — while we were all keen to find out how our students had fared, we knew there was hard work ahead. Richard and I picked up our scripts that evening and had a preliminary look at them. We were encouraged to see that Galin, Anthony, Derek and Stephen had all made progress on Problem 1. Closer inspection would reveal that Anthony and Stephen had solved the problem, that Galin had included most of the key points but had an error in his reasoning, and that Derek had made the first crucial step, worth three points in the marking scheme. Unfortunately for Derek the second half of his solution contained an arithmetic error that made the desired conclusion appear to fall out very easily; no doubt he thought he had solved the problem and did not return to it.

Although none of our students had solved Problem 2, Krzysztof, Derek, Stephen and Jamie had all described a crucial example identifying the correct answer to the problem. This was worth 1 of 7 points in the marking scheme for Problem 2. Unfortunately we could find nothing in our students' efforts on Problem 3 to inspire us to argue for a point on this very difficult problem.

Wednesday began with the assembly of the jury for the question and answer session on the second paper. Again the number of questions was fairly low and they were handled without fuss. One of the Irish students asked a two-part question on Problem 6, which fortunately could be answered concisely with the words "yes" and "no". After the questions and answers Richard and I resumed our study of 
the first day's scripts, going through them carefully now and in full detail.

A jury meeting was scheduled for after lunch, its purpose being the election of the president and two members of the IMO Advisory Board. The competition for the presidency was between the Hungarian leader and incumbent president, József Pelikán, and the Russian leader Nazar Agakhanov. The vote was won by József Pelikán. Myung Hwan Kim of Korea and Patricia Fauring of Argentina were then elected as members of the Advisory Board. I was disappointed for Geoff Smith who narrowly missed out on election and in my opinion has very progressive ideas on the future running of the IMO. However the successful candidates will undoubtedly do a fine job.

It was time now for the leaders, deputy leaders and observers to get together for coordination. This year it was the deputies who moved, and they arrived in Portorož on Thursday afternoon. It was great to see Gordon and get the news from Ljubljana and the students. While absorbed in the sheltered and relatively civilised business of problem selection and endless jury meetings, it is easy to forget that there is a larger and much more important side to the whole production at another location, some miles away and involving the actual participants in the event. Gordon reported that the Irish students were content after the two papers - Stephen had spent a lot of time on Problem 6 and believed he had solved it; Gordon was sceptical and alas his doubts turned out to be well-founded. The sociable atmosphere that followed the deputy leaders' arrival was soon interrupted by news that the second batch of scripts were awaiting collection. We set to work again.

It was immediately clear that making sense of the Day 2 scripts would present more of a challenge than those from Day 1. Our students had written a lot of material on Problem 4, and while it was clear that they had made some headway with it, it was equally clear that brevity was not a feature of any of their possible solutions. Stephen had written quite a few pages on Problem 6 and had told Gordon that he had solved this problem.

Galin had solved Problem 4 in a fairly straightforward manner and mercifully had tried to guide us through his numerous pages by highlighting the relevant parts and labelling the irrelevant parts as such. Krzysztof had begun with a suitable factorisation and launched into an analysis of cases as expected. Although he successfully dealt with 
some of the cases we regretfully concluded that his argument was not complete, nor could it be completed without significant extra work. Anthony, Derek and Stephen did not make great progress towards solving the problem, but each of them would pick up a point or two for writing down a useful factorisation or for spotting the solutions of the diophantine equation. Jamie had divided the problem up into far more cases than necessary and proceeded to analyse them separately in a long narrative. The three of us read this in turn, carefully and painstakingly, and we eventually satisfied ourselves that he had solved the problem. Satisfying the coordinators would be another matter as they could hardly be expected to have gone through this solution in the detail necessary to verify its correctness. Jamie's solution concluded with the line "Having exhausted all the possibilities, I now ...", and Richard aptly commented that the possibilities were not all he exhausted.

None of our students had made much progress on Problem 5 but in the middle of his effort Derek had vaguely intimated that the polynomial $Q(x)$ permutes the integer fixed points of $P(x)$. Since a clear statement to this effect was worth a point, we decided to ask for one.

On Problem 6 only Stephen had made a substantial effort, and unfortunately his attempt at solving the problem was based on a hunch that was simply not true. Nothing could be salvaged. It was a great pity for Stephen that he spent so much time on Problem 6 to no avail; he probably would have made progress on Problem 4 had he spent more time on it, and got partial or full marks. Partial credit is always very difficult to pick up on the hard problems, especially Problem 6.

\section{Coordination}

Coordination is the process through which marks are officially assigned. The chief coordinator for each problem oversees the team of coordinators for that problem, who are mathematicians. The representatives of each country have a half-hour meeting scheduled with a pair of coordinators for each problem, during which time agreement is usually reached on marks for that country's contestants. In most cases the marking scheme is decisive, everything is clear cut, and agreement is quickly reached. However if a dispute arises it can be quite difficult to resolve. 
There were four coordination tables allocated to each problem, each staffed by two coordinators. Since 90 countries participated, this meant that each pair of coordinators had to assess the efforts of well over 100 students, an extremely onerous task. In case anything in a student's work is not immediately clear, the representatives of that student's country need to be able to point out in a succinct manner at the coordination meeting exactly what the student is saying and why he or she deserves marks according to the marking scheme. Coordinators cannot award marks until they are fully satisfied that they are merited. Thus leaders, deputies and observers need to prepare carefully for coordination meetings in order to present their students' work fairly.

The coordination process in Slovenia was expertly managed. The coordination schedule was distributed on Thursday evening, and throughout the process the current status was prominently displayed on a large screen which was constantly updated. This showed which coordinators were waiting for which countries, and also showed any tables that were running ahead of schedule and were temporarily free, in which case a country could jump in out of turn and expedite the whole process. The coordinators in Slovenia did an excellent job in my opinion. All the coordinators that we dealt with were courteous, knowledgeable and more than willing to listen to our representations on behalf of our students. There was trouble over the coordination of Problem 2 (which as it happens did not affect us) but that was the only major glitch.

On Problem 1 we were able to agree on 7 for both Anthony and Stephen. This took a small effort in the case of Stephen as the end of his solution involved an longish and confusing digression to rule out a certain configuration that could obviously not arise, and of which explicit mention was not required. Galin ended up with 5 on this problem which we all agreed was fair, as his solution contained most of the required ingredients but also a clear error in reasoning. Derek's score was a clear 3 from the marking scheme.

Coordination of Problem 2 went exactly as expected, with a point each for Krzysztof, Derek, Stephen and Jamie for describing an optimal configuration. It was similarly quick matter to sign off on six zeroes for Problem 3. Since Problem 3 was an Irish problem, proposed by Finbarr Holland, it fell to us to coordinate this problem for the Slovenian team. The IMO convention is that the host country's marks are not assigned by local coordinators, but each problem 
is coordinated instead by the leaders from the country which proposed the problem. Unfortunately for the Slovenians their scores on Problem 3 were the same as ours.

Coordination of Problem 4 was a slightly more complicated procedure. We were able to highlight the main points in Galin's solution and agree on 7 fairly quickly. The discussion of Jamie's lengthy solution took a little longer but again we agreed on 7 . We agreed on a score of 3 for Krzysztof who had argued along appropriate lines but not been able to complete a proof. Our other team members also picked up some points on this problem. On Problem 5, we were pleased to discover that the coordinators had also spotted the relevant note in Derek's solution and were willing to award a point before we even asked for it. When we arrived at the coordination table for Problem 6, the coordinators told us that they had so far awarded a total of two points to a total of 19 countries. Nothing happened at our meeting to buck this trend.

As the scores rolled in they were being posted on huge notice boards, and as usual developments were carefully observed by Gordon, who is famous for being the first person in the world to know where the medal cut-offs will be. IMO rules stipulate that no more than marginally more than half of all students should receive medals, and that gold, silver and bronze medals should be awarded in a ratio as near as possible to 1:2:3. As the notice boards filled up it became apparent that the two hard problems had proved to be very hard indeed. On Problem 3 only 29 of 498 contestants achieved full marks, and only 133 were awarded any marks at all. (Of these 133, 95 scored only 1 point, presumably for factorising the left hand side of the inequality in the problem). This was surprising to many leaders who had expected students to do much better on the problem, particularly after it had been observed that it could be solved using Lagrange Multipliers after a non-obvious change of variables. This had in fact led to calls from some jury members for discarding the problem at the last moment. Problem 6 proved even more difficult, with only 27 students scoring any points and only 8 students achieving full marks.

By contrast, the two easy problems did prove easy, with 358 students solving Problem 1, 248 solving Problem 4 and many more scoring well on both. The medal cutoffs would be decided by performance on the two medium problems. 
Coordination of most problems was completed ahead of schedule by mid-morning on Saturday. A final jury meeting whose business would include agreement on medal cutoffs was scheduled for Saturday night. Coordination of Problem 2 however was proving problematic and continued well into Saturday evening. Many leaders felt that the coordinators were extremely reluctant to award partial marks on this problem and were heavily penalising minor omissions in essentially correct proofs. This led to delays and general disgruntlement, with some leaders eventually settling to accept marks that they perceived to be unfairly low, in the knowledge that many others were in the same boat. In the event of failure to reach agreement with coordinators a leader may ask the jury at the final meeting to adjudicate. This happened in the case of two countries in Slovenia, both disputes involving Problem 2. Since many leaders felt that they had conceded in similar disagreements, the jury voted to accept the judgement of the coordinators in both cases. The difficulty over Problem 2 was the major negative feature of an otherwise excellent coordination process.

Medal cutoffs were set at 15 for bronze, 19 for silver and 28 for gold. This meant that 253 of the 498 contestants were awarded medals. There were three perfect scores, by Zhiyu Lu of China, Alexander Magazinov of Russia and Iurie Boreico of Moldova. Although the IMO is a contest for individuals, there is an unofficial tally kept of each country's overall performance. This year's winner was China, with six gold medals and a total of 214 points (from a maximum of 252). Ireland finished in 67th place.

We departed Portorož early on Sunday morning in a fleet of coaches. We would visit Bled and the Julian Alps before arriving in Ljubljana where the closing ceremony and presentation of medals would take place on Monday. We met up with the contestants in the town of Kranjska Gora in the spectacular Zgornjesavska Valley, near the Austrian border. Our students were in good form although Stephen was a little disappointed with his score on Problem 6. There was a general air of festivity, particularly among the students, who had completely switched to holiday mode since the completion of their hard work three days earlier.

Richard left for Ireland on Monday morning as he had commitments there and would unfortunately miss the closing ceremony. We had some time for sightseeing in Ljubljana that morning, and I decided to skip the guided tour and explore the streets alone instead. 
Ljubljana has all the grand architecture that one would expect in a European capital, but it is not a very large city and has a very pleasant unhurried atmosphere.

We convened at the huge auditorium of Cankarjev Dom for the closing ceremony, and Gordon and I were pleased to find seats with our students. The ceremony proceeded with some short speeches, a performance of traditional music and dance, and the presentation of medals. The names of those students who were awarded an honourable mention, including Galin, Anthony, Stephen and Jamie, were listed on the screen. The ceremony ended with the passing on of the IMO banner to the Vietnamese, who will host the 48th IMO in 2007. Matching the efficiency, style and good humour with which this year's IMO was staged by the Slovenians will be a major challenge, but no doubt they will be up to it.

\section{REMARKS}

In my opinion there is much to be optimistic about following Ireland's participation in this year's IMO. Our team finished with 49 points and four honourable mentions. This is a more balanced performance than last year, when 34 of the overall 55 points were accounted for by a single competitor, in the best ever performance by a contestant representing Ireland at an IMO. We had a young team this year; most of its members remain eligible at least for next year. We can certainly hope for medals in the next couple of years if the 2006 performance can be built on.

On the other hand I think that we need to look critically at how it can be built on. Irish participation in the IMO is managed by a small group of dedicated volunteers who are academic staff members in third level institutions. These volunteers do an excellent job with limited resources. They run training programmes and oversee the selection and preparation of the Irish team. However most second level students in Ireland probably have no awareness of the IMO or Ireland's participation in it. Students are invited to the training through the results of the Junior Certificate Examination and to an extent through word of mouth. Many other countries hold preliminary rounds in their national olympiads in which huge numbers of students participate and in which their schools are closely involved. I believe that this is a suitable time to introduce such a contest in Ireland. There are at least two reasons to do so - one is 
that every Irish second level student would have the opportunity to be involved in the process leading to IMO participation. Students would be exposed to problem-solving contests at an early age, and in all likelihood interested and talented students would be identified. Another reason to hold such a contest is that it would create a natural way for teachers at second level to become involved in the IMO and the activities that surround it. It would open a channel of communication between educators at second and third levels with an interest in mathematics enrichment, and create opportunities for the establishment of a wider support network for mathematically inclined students.

I also wonder if perhaps we should make more demands of students in our mathematical enrichment/IMO training programmes. Maybe a more rigourous programme of practice problems that students are expected to work on and hand up is something we could consider. I am not familiar with practices at all the Irish training centres, but I believe that here at NUI Galway we could do more to convey to students that they will improve their skills only through independent work, and that attendance at training sessions is only a tiny part of what is required to become adept at problem-solving. We certainly provide practice problems and encourage students to work on them and send us their efforts, but we don't insist that they do so. As anybody reading this document is likely to know, the most successful mathematical olympian to represent Ireland so far is Fiachra Knox, who narrowly missed a gold medal in Mexico last year, and attained a bronze medal in Greece the previous year. Fiachra's success can of course be partly attributed to his phenomenal talent, but his improvement from one year to the next was due in no small part to the fact that following the 2004 IMO he immersed himself in problem-solving. He gratefully accepted the help and advice offered to him from various quarters but he also took control of his own progress. I am not suggesting that we insist that prospective IMO participants should become obsessed with problem solving to the exclusion of all else, but I wonder if perhaps our approach is a little too uncompetitive and unstructured at present. Significant personal resources are being invested by volunteer trainers, students and others in the training effort, and it is in everybody's interest to try to optimise the return on this investment.

An interesting aspect of this year's event from an Irish point of view is the fact that Slovenia (with a population of approximately 2 
million) is the smallest country ever to have hosted an IMO. Moreover there was a general consensus that the Slovenian organisers did an outstanding job. Everything ran extremely efficiently, and it was clear that the practical and technical preparations were top-class. This is evident even from looking at the excellent event website at http://imo2006.dmfa.si. Indeed there were other examples during the IMO of administrative processes being effectively backed-up by careful preparation, such as the management of students' queries from the exam hall, and the electronic handling of translations of the problems into various languages. Having attended the 47th IMO in Slovenia I have no doubt that Ireland possesses the necessary infrastructure to host an IMO. The Slovenians were very congenial hosts and I hope we can match them there; matching their frightening organisational efficiency will be a challenge but I hope we could try.

During our stay in Slovenia, Richard and I informally discussed the possibility of Ireland hosting an IMO with members of the IMO Advisory Board and with leaders from some other countries. Our impression is that a formal proposal from Ireland would be welcomed with great enthusiasm by the general IMO community. A suitable year might be 2013, which will be the 25th anniversary of Ireland's first IMO participation. Of course there would be difficulties, availability of funds being one and availability of suitably qualified and capable coordinators in sufficient numbers being possibly another. However the Slovenians certainly faced this second problem and handled it by inviting some colleagues from neighbouring countries to act as coordinators. I am sure that we would also find neighbours willing and able to help if necessary. I know that there is significant support in the Irish mathematical community for the idea of proposing to host the $2013 \mathrm{IMO}$; it is time now to conduct a serious feasibility study and estimate of costs. It would be wonderful if Ireland could be in a position to make an official statement in Vietnam next year.

\section{ACKNOWLEDGEMENTS}

There are many people, in Slovenia and Ireland, who contributed valuably to the success of the Irish team at the IMO this year. Thanks firstly to the organisers of the event for doing such an excellent job and for making the experience so enjoyable for all participants. Thanks also to all the IMO staff at both Portorož and Ljubljana, especially the Irish team's guide Nejc Habjan. 
I would like to thank all those who participated in the mathematical enrichment programmes in the five Irish training centres this year. These include all the students who took part in the training, and their teachers and parents, who in many cases undertook to drive long distances at inconvenient times. I would particularly like to thank the coaches at the training centres who voluntarily gave of their time and expertise to expose our most talented students to new mathematical ideas. This year's trainers were

- At UCC: Jerry Buckley, Rory Conboye, Prithwijit De, Richard Ellard, Finbarr Holland, Donal Hurley, Seamus O'Ceallaigh, Ricky O'Riordan, Dmitrii Rachinskii and Thomas Ralph.

- At UCD: Mark Dukes, Mark Flanagan, Kevin Hutchinson and Tom Laffey.

- At UL: Mark Burke, Bernd Kreussler, Jim Leahy and Gordon Lessells.

- At NUI Galway: James Cruickshank, Niall Madden, Götz Pfeiffer, Rachel Quinlan, Emil Sköldberg, Jerome Sheahan and James Ward.

- At NUI Maynooth: Stephen Buckley, Kurt Falk, Pat McCarthy, Gary McGuire, John Murray, Tony O'Farrell, Adam Ralph, José Ramón Marí, David Redmond, Ian Short, Anthony Small and Richard Watson.

Thanks also to the above named universities for their provision of training facilities, especially to the University of Limerick for their continued support and hosting of the pre-olympiad training camp. Special thanks on that note to Gordon Lessells for his continued organisation of the Limerick camp, and to Mark Flanagan and Fiachra Knox for their participation in this year's camp. Thanks to all the people who proposed problems for this year's Irish Mathematical Olympiad and IMO, especially to Finbarr Holland whose problem was short-listed and selected as a contest problem. On a personal note I would like to thank other recent Irish leaders for their guidance and advice, especially James Cruickshank and Tom Laffey.

Ireland could not participate in the International Mathematical Olympiad without the continued financial support of the Department of Education and Science, which is gratefully acknowledged. Particular thanks to Doreen McMorris, chairperson of the Irish Olympiad 
Participation Committee. Thanks also to the Irish Mathematical Society and to the Mathematical Sciences subcommittee of the Royal Irish Academy for their support.

Finally I would like to thank the six members of this year's Irish team for their hard work and dedication to the task. I hope that their experience at the 47th IMO will inspire them to reach for new mathematical heights.

Rachel Quinlan,

Department of Mathematics,

NUI Galway,

Galway, Ireland

rachel.quinlan@nuigalway.ie

Received on 13 December 2006. 